\title{
Assessments of Pudendal Motor and Sensory Nerves in Patients with or without Fecal Incontinence after Low Anterior Resection for Lower Rectal Cancer
}

\author{
Ryouichi Tomita ${ }^{1,2}$, Sakurai Kenichi1,2, Shigeru Fujisaki1,2 \\ ${ }^{1}$ Department of Surgery, School of Life Dentistry, Nippon Dental University, Tokyo, Japan \\ ${ }^{2}$ First Department of Surgery, School of Medicine, Nihon University, Tokyo, Japan \\ Email: ^rtomita@tky.ndu.ac.jp
}

How to cite this paper: Tomita, R., Kenichi, S. and Fujisaki, S. (2020) Assessments of Pudendal Motor and Sensory Nerves in Patients with or without Fecal Incontinence after Low Anterior Resection for Lower Rectal Cancer. Journal of Cancer Therapy, 11, 220-235.

https://doi.org/10.4236/jct.2020.114019

Received: March 27, 2020

Accepted: April 19, 2020

Published: April 22, 2020

Copyright $\odot 2020$ by author(s) and Scientific Research Publishing Inc. This work is licensed under the Creative Commons Attribution International License (CC BY 4.0).

http://creativecommons.org/licenses/by/4.0/

\begin{abstract}
Background: To clarify the pudendal motor (PMN) and sensory (PSN) nerves play in preventing fecal incontinence (FI) after low anterior resection (LAR) for lower rectal cancer, the PMN and PSN functions were studied. Methods: Sixty patients were divided into groups A $(n=20, F I)$ and B $(n=40$, continence). These were compared with group $C(n=30$, control subjects). PMN latency (PMNL) (right, left, and posterior sides of the anal canal) was studied by sacral magnetic stimulation. Anal mucosal electric sensitivity (AMES) was measured at the lower, dentate line (DL), and upper zones. Results: The distance of anastomosis from anal verge (DAAV) in group A was significantly shorter than in group B ( $p$ value $<0.001)$. In PMNL, the conduction delay in group A was significantly longer than in groups $\mathrm{B}$ and $\mathrm{C}$ at all sides ( $p$ value $<$ 0.001 , respectively). On AMES, the sensitivity of patients in group A was significantly lower than in groups $\mathrm{B}$ and $\mathrm{C}$ at all zones ( $p$ value $<0.001$, respectively). In groups $\mathrm{A}, \mathrm{B}$, and $\mathrm{C}$, correlation coefficients between SMNL (posterior side) and AMES (DL zone) were $0.780,0.858$, and 0.793 , respectively ( $p$ value $<0.001)$. Conclusion: FI after LAR with a short DAAV may lead to external anal sphincter dysfunction due to damage of both PMN and PSN.
\end{abstract}

\section{Keywords}

Low Anterior Resection, Lower Rectal Cancer, Pudendal Motor Nerve, Pudendal Sensory Nerve, Fecal Incontinence

\section{Introduction}

In low anterior resection (LAR) for lower rectal cancers (LRC), $20.0 \%$ to $40.0 \%$ 
of postoperative patients after LAR experience soiling [i.e. minor fecal incontinence (FI)] and/or FI (i.e. involuntary defecation), reducing the postoperative quality of life (QOL) even 1 to 2 years after LAR [1] [2] [3]. Various dysfunctions as causative factors, such as sphincter muscle function, rectal retention ability, and recto-anal inhibitory reflux, have been mainly documented based on manometric studies [4] [5] [6]. Of these factors, sphincter dysfunction has been focused on as the main cause. The external anal sphincter (EAS), internal anal sphincter (IAS), and puborectalis muscle (PM) essentially establish continence [5] [7] [8]. Among these sphincter muscles, EAS is the most important to prevent FI [5] [7] [8]. Especially, voluntary contractions of EAS prevent FI that may be induced by an increase in abdominal pressure due to coughing or postural changes [7] [8].

Regarding nerve control of EAS, investigations have mainly focused on EAS regulation by the pudendal motor nerve (PMN) [4] [9] [10] [11]. To date, measurement of the PMN terminal latency has involved direct electrical stimulation of the terminal PMN in the anal canal [9] [10] [11] [12]. There have been many reports of findings obtained on straining during defecation in patients with chronic constipation [13] [14]. It is well-known that PMN extension injury caused by over-extension of the pelvic floor muscle due to straining during defecation promotes FI [13] [14]. Thus, an impaired regulation of EAS by damaged PMN may lead to FI. Regarding studies on PMN terminal latency in LAR patients with soiling or FI, its delay has been suggested [11] [12].

Moreover, attenuation of the anal mucosal sensitivity mediated by the pudendal sensory nerve (PSN) to discriminate gas and feces from other contents has also been documented in patients with FI [14] [15] [16] [17]. Previously, the author reported that impairment of the anal canal mucosal electrical sensitivity (AMES) threshold in LAR patients may be directly associated with soiling [16].

Until now, PMN and PSN functions in patients after LAR have been studied in different patient groups [9] [10] [11] [12] [16] [18] [19]. In this study, both the PMN and PSN functions were studied in an identical patient group with or without FI after LAR for RC. Moreover, measurement of the pudendal motor nerve latency (PMNL) by sacral magnetic stimulation (SMS) is still not applied for evaluations of LAR patients with or without FI. So, the author used SMS to evaluate the PMN function in LAR patients with or without FI in the present study.

By the way, in patients after LAR at an early postoperative status, less than 6 months after LAR, there is no report of the PMN and PSN functions for FI. Therefore, the authors performed this study.

\section{Patients and Methods}

\subsection{Patients}

From January 2005 to December 2015, a total of 60 patients aged 44 to 78 years (40 men and 20 women with a mean age of 62.6 years) who underwent LAR for LRC were enrolled in the present study. Based on postoperative FI, these patients 
were divided into 2 groups [group A, patients with FI $(\mathrm{n}=20), 17$ men and 3 women aged 44 to 78 years with a mean age of 63.3 years; group B, patients without FI (continence, $n=40$ ), 23 men and 17 women aged 47 to 77 years with a mean age of 61.9 years]. Groups A and B were compared with control subjects [group C, 30 volunteers with normal bowel movements (20 men and 10 women aged 46 to 76 years with a mean age of 60.1 years).

Stage groupings were used for TNM classification. All patients were pathological Stage I (44 patients: T1, N0, M0; 16 patients: T2, N0, M0)]. The patients underwent straight anastomosis, and the distance of the anastomosis from the anal verge (DAAV) was 2 to $6 \mathrm{~cm}$ based on an anorectal scope. No recurrence was noted at the time of examination.

Subjects were without surgical histories, psychiatric diseases, metabolic-endocrine disorders, digestive tract impairments, defecation abnormalities, and chronic constipation. Furthermore, all female subjects were nulliparous.

This retrospective study was performed at 14 months after LAR. Based on the Wexner score [20] (Table 1), the author assessed their stool status directly. FI in group A was defined as 6 or higher, while fecal continence in group B was defined as 5 or lower. Then, LAR patients could be divided into those with (group A) and without (group B) patients. The author was not blinded regarding whether patients were continent or incontinent.

To clarify FI from the function of SMN and PSN, a homogeneous patient group was targeted for this preliminary study. The author considered that the analysis of data would be straightforward if the patient characteristics were homogenous.

\subsection{Surgical Procedure}

In all patients, the flexure of the spleen was displaced to allow the descending colon to reach the pelvic floor without tension. All patients were UCC Stage I in the present study. Then, the sigmoid colon and rectum were resected and total mesorectal excision with regional lymph node resection while preserving autonomic nerves, such as bilateral hypogastric nerves and pelvic plexuses, were performed in all patients. The distal rectum was closed with a ROTICULATOR 55 instrument (United States Surgical, Norwalk, CT, USA). Straight anastomosis between the descending colon and remnant rectum was performed using the PREMIUM CEEA instrument (United States Surgical).

Covering the stoma was not performed in any of the LAR patients.

\subsection{Measurement of PMNL by SMS}

PMN derived from the S2-4 spinal nerve roots innervating the EAS can be excited transcutaneously by stimulating the spinal column overlying the cauda equina, allowing measurement of the motor latency to this muscle. The patient was placed in the left lateral position and a separate ground electrode was connected from the upper thigh to the preamplifier of the electromyography (EMG) apparatus (Isolator SS 104J, 7T18 Signal Processor, Sanei-Nihonkoden, Tokyo, 
Table 1. The Wexner score (a frequency assessment tool).

\begin{tabular}{cccccc}
\hline Type of incontinence & Never & Rarely & Sometimes & Usually & Always \\
\hline Solid & 0 & 1 & 2 & 3 & 4 \\
Liquid & 0 & 1 & 2 & 3 & 4 \\
Gas & 0 & 1 & 2 & 3 & 4 \\
Wear pad & 0 & 1 & 2 & 3 & 4 \\
Lifestyle altered & 0 & 1 & 2 & 3 & 4 \\
\hline
\end{tabular}

Never: 0; Rarely: Less than once a month; Sometimes: Less than once a week or once a month; Usually: Once a day or once a week; Always: Once a day or more.

Japan). The stimulus was applied from a magnetic stimulator (Magstim-Model 200, Nihonkoden, Tokyo, Japan), consisting of a flat coil with an outer diameter of $9 \mathrm{~cm}$ placed in the area overlying S2-4. A large, brief pulse of current (peak value: $4000 \mathrm{~A}$ after $110 \mu \mathrm{s}$ ) was then passed from a high-voltage capacitor discharge system through the coil. The EAS response was recorded with a glove-mounted electrode array. This consisted of two metal plates mounted $1 \mathrm{~cm}$ apart at the tip of the gloved index finger. The finger bearing this device was inserted into the anal canal so that the electrode array was in contact with EAS on the right, left, and posterior sides. Magnetic stimulation at the S2-4 sacral levels has been shown to activate the PMN root of the cauda equine. The latency of induced EMG activity ( $M$ wave: motor activity potential evoked from EAS muscles directly regulated by the stimulated PMN derived from the S2-4 spinal nerve roots) of EAS is the value of PMNL (see Ref. [9] [10] [11] [12] [16] [18] [19]). Contraction of EAS could be easily detected by index finger insertion into the anal canal.

\subsection{Measurement of AMES Threshold}

A ring-type bipolar electrode, with 2-mm-wide platinum electrodes fixed at 1 and $1.7 \mathrm{~cm}$ from the tip of a 12-Fr silicon Foley catheter (Ballon Catheter type 94001; Unique Medical, Tokyo, Japan), was devised in our laboratory. During monitoring, the subject assumed a left lateral decubitus position, and DL was confirmed by an anorectal scope before inserting the catheter through the anus. A total of 3 zones with reference to DL were categorized according to the lower end of the stimulation electrode: upper (the region at $1 \mathrm{~cm}$ on the oral side from $\mathrm{DL}$ ), middle (the DL region), and lower (the region at $1 \mathrm{~cm}$ on the anal side from DL) levels in the anal canal. Under stimulation conditions, currents of rectangular waves with a $0.2-\mathrm{ms}$ duration and gradually increasing intensity (starting from 0.1 to peaking at $30 \mathrm{~mA}$ ) were delivered at a $1-\mathrm{Hz}$ frequency using a constant current generator (Electric Stimulator SMN-7203; Nippon-Koden, Tokyo, Japan) (see ref. [16]). The current (in $\mathrm{mA}$ ) that induced a tingling/pulsing sensation in the patient was defined as the AMES threshold.

\subsection{Experimental Procedure}

The above two tests were conducted in a shielded room at $25^{\circ} \mathrm{C}$. No bowel prep- 
aration was performed, and after an overnight fast patients were given the opportunity to defecate before the experiment. Also, patients did not receive breakfast, dietary restrictions, a glycerin-enema, or drug pretreatments. No sedative was used. Measurements were conducted at 9:00am with patients in the left lateral decubitus position and knees raised to the chest.

It was necessary for the author to measure the PMNL values and AMES threshold in the present study. For this, the author needed to obtain informed consent from subjects participating in this study. To assess of FI after LAR, the author also obtained corporation from each patient after LAR and control subjects.

Application of PMNL by the SMS method and measurements of the anal mucosal electrical sensitivity (AMES) threshold in the present study were approved by the Ethical Committee of Nihon University School of Medicine.

\subsection{Statistical Analyses}

Statistical analyses were conducted using the non-parametric Kruskal-Wallis test with Bonferroni correction (StateView version 5.0 for Macintosh; Abacus Concepts Inc., Berkeley, CA, USA). Results are presented as the mean \pm standard deviation (SD). For statistical analysis of the characteristics of patients, the chi-squared test (2-tailed test) was used. Data were analyzed, and the DAAV was calculated using Student's t-test. Correlations between SMNL and AMES were calculated by Spearman's rank correlation analysis. A $p$ value of less than 0.05 was regarded as significant.

\section{Results}

\subsection{Patient Characteristics}

There were no significant differences in patients' characteristics between groups $A$ and B except for sex and DAAV. Group A had a significantly larger proportion of males than group $B$ ( $p$ value $=0.033$ ). In addition, there are no significant differences between the demographic data in groups $\mathrm{A}, \mathrm{B}$, and C.

As for DAAV, groups A showed significantly shorter distances than group $\mathrm{B}$ ( $p$ value $<0.001)$ (Table 2$)$.

\subsection{The FI Score (Wexner Score) after LAR}

FI after LAR was evaluated by the Wexner score [20] (Table 1). Wexner scores from 8 to 10 (mean: 9.25) comprised 20\% of group A, 11 to 15 (mean: 13.5) $50.0 \%$, and 16 to 20 (mean: 18.5) comprised 30.0\%. All patients in group A were incontinent. In contrast, all patients in groups B (Wexner score: 0 ) and $\mathrm{C}$ (Wexner score: 0) were continent. Patients in pre-operative defecation (Wexner score; 0 ) were also continent (Table 3 ).

\subsection{Values of PMNL}

Right side: Group A exhibited significant prolongations compared with groups B and $\mathrm{C}$ ( $p$ value $<0.001$, respectively). There was no significant difference be- 
tween groups $\mathrm{B}$ and $\mathrm{C}(p$ value $=0.104)$. Left side: Similar to the right side, group A displayed significant prolongations compared with groups $\mathrm{B}$ and $\mathrm{C}$ ( $p$ value $<0.001$, respectively). There was no significant difference between groups $\mathrm{B}$ and $\mathrm{C}$ ( $p$ value $=0.146)$. Posterior side: group A exhibited significant prolongations compared with groups B and C ( $p$ value $<0.001$, respectively). There was no significant difference between groups B and C $(p$ value $=0.174)($ Table 4$)$.

Table 2. Characteristics of patients.

\begin{tabular}{|c|c|c|c|}
\hline Factors & Group A $(n=20)$ & Group B $(\mathrm{n}=40)$ & $p$ value \\
\hline Male/Female & $17 / 3$ & $23 / 17$ & 0.033 \\
\hline Age (years) & $63.3(44-78)$ & $61.9(47-77)$ & ns \\
\hline \multicolumn{4}{|c|}{ Depth of cancer invasion } \\
\hline $\mathrm{T} 1(\mathrm{n}=44)$ & $70.0 \%(14 / 20)$ & $75.0 \%(30 / 40)$ & \multirow[b]{2}{*}{ ns } \\
\hline $\mathrm{T} 2(\mathrm{n}=16)$ & $30.0 \%(6 / 20)$ & $25.0 \%(10 / 40)$ & \\
\hline \multicolumn{4}{|c|}{ Lymph node metastasis } \\
\hline No & $100 \%(20 / 20)$ & $100 \%(40 / 40)$ & ns \\
\hline \multicolumn{4}{|c|}{ Pathological stage } \\
\hline Stage I & $100 \%(20 / 20)$ & $100 \%(40 / 40)$ & ns \\
\hline \multicolumn{4}{|c|}{ Preservation of bilateral hypogastric nerves and pelvic plexuses } \\
\hline Preservation & $100 \%(20 / 20)$ & $100 \%(40 / 40)$ & ns \\
\hline \multicolumn{4}{|c|}{ Level of anastomosis from the dentate line $(\mathrm{cm})$} \\
\hline & $2.4 \pm 1.7 \mathrm{~cm}$ & $4.3 \pm 0.9 \mathrm{~cm}$ & $<0.001$ \\
\hline & $(2-4)$ & $(3-6)$ & \\
\hline \multicolumn{4}{|l|}{ Past history } \\
\hline No & $100 \%(20 / 20)$ & $100 \%(40 / 40)$ & ns \\
\hline \multicolumn{4}{|c|}{ Postoperative complications } \\
\hline Wound infection & $10 \%(2 / 20)$ & $10 \%(4 / 40)$ & ns \\
\hline
\end{tabular}

Group A: Patients withfecal incontinence after low anterior resection; Group B: Patients withoutfecal incontinence after low anterior resection; ns: No significance.

Table 3. The fecal incontinence score after LAR.

\begin{tabular}{ccccc}
\hline & $\begin{array}{c}\text { Pre-operative } \\
(\mathrm{n}=60)\end{array}$ & $\begin{array}{c}\text { Group A } \\
(\mathrm{n}=20)\end{array}$ & $\begin{array}{c}\text { Group B } \\
(\mathrm{n}=40)\end{array}$ & $\begin{array}{c}\text { Group C } \\
(\mathrm{n}=30)\end{array}$ \\
\hline Distribution of Wexner scores & & & & \\
0 to 5 (good) & $60(100 \%)$ & $0(0 \%)$ & $40(100 \%)$ & $30(100 \%)$ \\
6 to 10 (fair) & $0(0 \%)$ & $4(20.0 \%)$ & $0(0 \%)$ & $0(0 \%)$ \\
11 to 15 (poor) & $0(0 \%)$ & $10(50.0 \%)$ & $0(0 \%)$ & $0(0 \%)$ \\
16 to 20 (very poor) & $0(0 \%)$ & $6(30.0 \%)$ & $0(0 \%)$ & $0(0 \%)$ \\
\hline
\end{tabular}

Group A: Patients with fecal incontinence after low anterior resection; Group B: Patients without fecal incontinence after low anterior resection; Group C: Control subjects. 
Table 4. Values of pudendal motor nerve latency.

\begin{tabular}{cccc}
\hline & Group A $(\mathrm{n}=20)$ & Group B $(\mathrm{n}=40)$ & Group C $(\mathrm{n}=30)$ \\
\hline Right side & $8.0 \pm 0.7 \mathrm{~ms}^{\mathrm{a}}$ & $4.2 \pm 0.5 \mathrm{~ms}^{\mathrm{b}}$ & $3.9 \pm 0.2 \mathrm{~ms}^{\mathrm{c}}$ \\
& $(6.0-9.8)$ & $(3.2-4.8)$ & $(3.4-4.2)$ \\
Left side & $8.1 \pm 2.7 \mathrm{~ms}^{\mathrm{d}}$ & $4.1 \pm 0.6 \mathrm{~ms}^{\mathrm{e}}$ & $3.8 \pm 0.3 \mathrm{~ms}^{\mathrm{f}}$ \\
& $(6.8-10.0)$ & $(3.2-4.9)$ & $(3.5-4.3)$ \\
Posterior side & $7.9 \pm 0.9 \mathrm{~ms}^{\mathrm{g}}$ & $4.1 \pm 0.6 \mathrm{~ms}^{\mathrm{h}}$ & $3.9 \pm 0.3 \mathrm{~ms}^{\mathrm{i}}$ \\
& $(5.9-9.0)$ & $(3.0-5.0)$ & $(3.4-4.4)$ \\
\hline
\end{tabular}

a vs. b: $p$ value $<0.001$, a vs. c: $p$ value $<0.001$, $\mathrm{b}$ vs. c: $p$ value $=0.104 ; \mathrm{d}$ vs. e: $p$ value $<0.001, \mathrm{~d}$ vs. f: $p$ value $<0.001$, e vs. f: $p$ value $=0.146$; g vs. $\mathrm{h}: p$ value $<0.001$, $\mathrm{g}$ vs. I: $p$ value $<0.001, \mathrm{~h}$ vs. I: $p$ value $=0.174$. Group A: Patients with fecal incontinence after low anterior resection; Group B: Patients without fecal incontinence after low anterior resection; Group C: Control subjects.

\subsection{Values of AMES Threshold}

The upper zone: Group A revealed a significant increase compared with groups $\mathrm{B}$ and $\mathrm{C}(p<0.001$, respectively). There was no significant difference between groups B and C ( $p$ value $=0.061)$. The middle zone: group A exhibited a significant increase in values compared with groups B and C ( $p$ value $<0.001$, respectively). There was no significant difference between groups B and $\mathrm{C}$ ( $p$ value $=$ 0.123). The lower zone: group A showed a significant increase in the threshold compared with groups B and C ( $p$ value $<0.001$, respectively). There was no significant difference between groups B and C ( $p$ value $=0.072)($ Table 5$)$.

In addition, LAR patients with FI showed a significant prolongation of PMNL values as well as a significant reduction of the AMES threshold compared with patients without FI. There were no significant differences in PMNL and AMES threshold values between LAR patients without FI and control subjects.

\subsection{Spearman's Rank Correlation Test between SMNL in the Posterior Wall and ASMES in the Middle Zone of the Anal Canal}

The posterior side of EAS is regulated by the bilateral sides of PMN. DL is well supplied with sensory terminals and organs, and exhibits the highest sensitivity in the anal canal. Therefore, the author conducted a correlation test between SMNL in the posterior wall and ASMES in the middle zone of the anal canal. Figure 1 shows regression lines that present the association of SMNL (sm) with AMES ( $\mathrm{mA}$ ) in groups A, B, and C. A high regression coefficient of more than 0.700 was noted in groups $\mathrm{A}, \mathrm{B}$, and $\mathrm{C}$. There were also significant differences in the correlation coefficient between SMNL and AMES in groups $\mathrm{A}, \mathrm{B}$, and $\mathrm{C}$ ( $p$ value $<0.001$, respectively).

\section{Discussion}

Generally, the Wexner score is the most widely used method to assess the efficacy of surgical therapies for FI [20]. Ito et al. [21] suggested that both poor (11 to 15 ) and very poor (16 to 20 ) scores in all patients after LAR indicated severe FI 
with solid and liquid stools. Koda et al. [22] reported that a Wexner score $\geq 10$ was a strong predictor of severe postoperative FI. Badic et al. [3] also suggested that a Wexner score of $\geq 10$ is an indication of FI. In this retrospective study, FI scores (Wexner score) in all patients with FI were 8 or more. Especially, FI scores of 10 or more indicated severe FI.

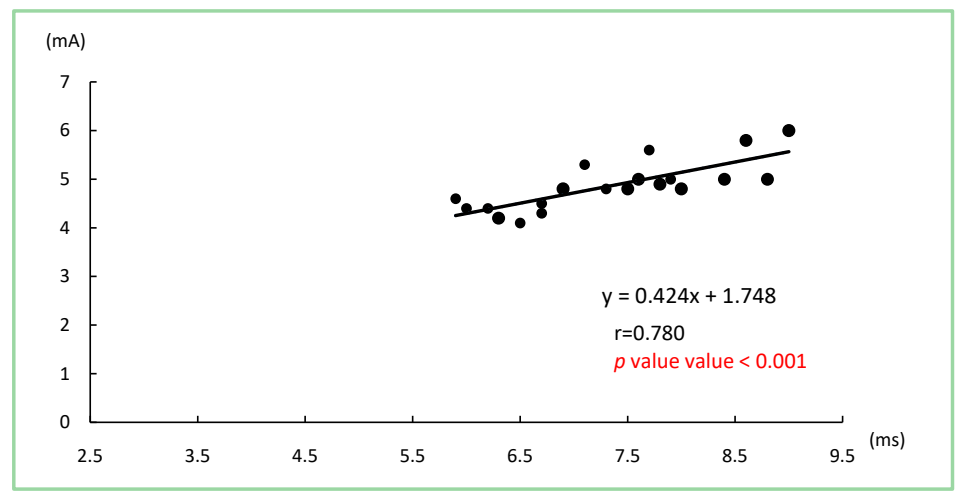

(a)

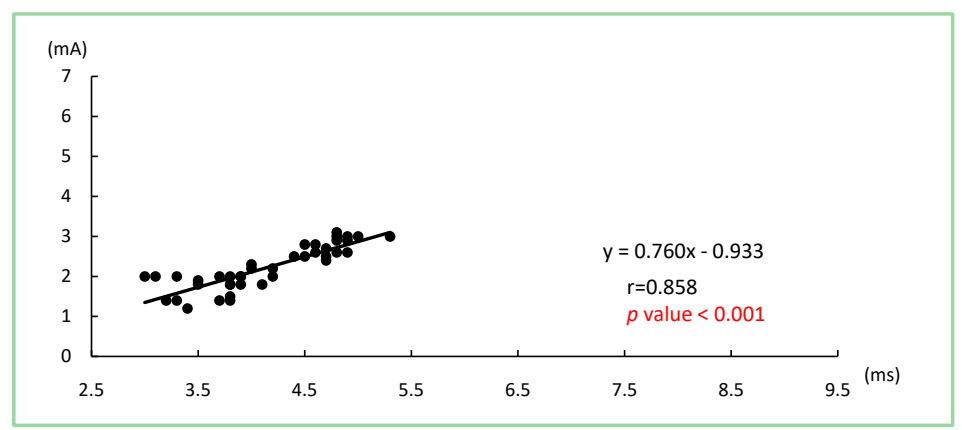

(b)

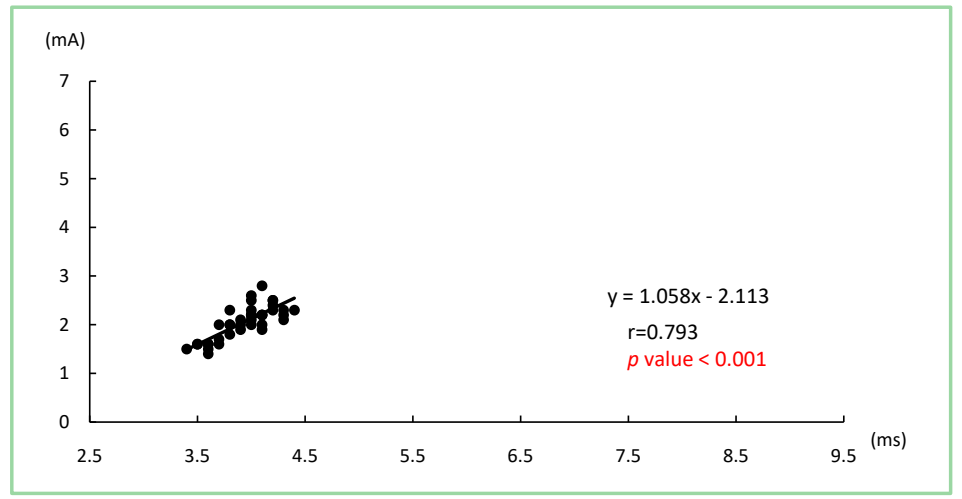

(c)

Figure 1. Regression lines in (a) (patients with incontinence after low anterior resection), (b) (patients without incontinence after low anterior resection, and (c) (control subjects). This figure shows regression lines that present the relation of the anal mucosal electric sensitivity threshold values (DL zone of the anal canal to those of pudendal motor nerve latency (SMNL: posterior side of the anal canal) in (a) (b) and (c). A high regression coefficient of more than 0.70 was shown. There were significant correlation coefficients between SMNL (posterior side of the anal canal) and AMES (DL zone of the anal canal) in groups $\mathrm{A}, \mathrm{B}$, and $\mathrm{C}$ ( $p$ value $<0.001$, respectively). 
Table 5. Values of anal mucosal electric sensitivity threshold.

\begin{tabular}{cccc}
\hline & Group A $(\mathrm{n}=20)$ & Group B $(\mathrm{n}=40)$ & Group C $(\mathrm{n}=30)$ \\
\hline Upper zone & $6.3 \pm 1.2 \mathrm{~mA}^{\mathrm{a}}$ & $2.6 \pm 0.6 \mathrm{~mA}^{\mathrm{b}}$ & $2.3 \pm 0.5 \mathrm{~mA}^{\mathrm{c}}$ \\
Middle zone & $(4.6-8.3)$ & $(1.5-3.8)$ & $(1.6-3.1)$ \\
& $5.0 \pm 0.5 \mathrm{~mA}^{\mathrm{d}}$ & $2.3 \pm 0.5 \mathrm{~mA}^{\mathrm{e}}$ & $2.1 \pm 0.4 \mathrm{~mA}^{\mathrm{f}}$ \\
Lower zone & $(4.2-5.8)$ & $(1.4-3.0)$ & $(1.4-2.8)$ \\
& $4.8 \pm 0.5 \mathrm{~mA}^{\mathrm{g}}$ & $2.5 \pm 0.6 \mathrm{~mA}^{\mathrm{h}}$ & $2.2 \pm 0.5 \mathrm{~mA}^{\mathrm{i}}$ \\
& $(4.1-6.0)$ & $(1.6-3.8)$ & $(1.6-2.8)$ \\
\hline
\end{tabular}

a vs. b: $p$ value $<0.001$, a vs. c: $p$ value $<0.001$, b vs. c: $p$ value $=0.061$; b vs. e: $p$ value $<0.001$, b vs. f: $p$ value $<0.001$, e vs. f: $p$ value $=0.123$; $\mathrm{g}$ vs. $\mathrm{h}: p$ value $<0.001, \mathrm{~g}$ vs. I: $p$ value $<0.001, \mathrm{~h}$ vs. I: $p$ value $=0.072$. Group A: Patients with fecal incontinence after low anterior resection; Group B: Patients without fecal incontinence after low anterior resection; Group C: Control subjects.

The functional outcome after LAR, especially FI, is closely related to DAAV [1] [2] [3] [7]. Generally, a remaining rectum length of $4 \mathrm{~cm}$ or more from the anal verge in patients after LAR while preserving autonomic nerves allows for a good anorectal function without FI [1] [2] [3] [7]. In the present study, DAAV in patients with FI (mean: $2.4 \mathrm{~cm}$ ) was significantly shorter than that in patients without FI (mean: $4.3 \mathrm{~cm}$ ).

Sphincter muscles including EAS, IAS, and PM are important for maintaining continence [7]. Generally, it is considered that EAS is the most important to prevent involuntary defecation compared with IAS and PM [4] [6] [8]. Even after IAS resection in patients after intersphincteric resection for RC, the anorectal function may improve over time [5] [8]. Andrius et al. [6] revealed that continence is affected by injury to the anal sphincter (mainly by EAS). It was reported that the EAS function recovered to preoperative levels after one year in patients receiving LAR without IAS preservation [19] [21]. EAS is also crucial for complementing accessory functions of feces retention/continence, i.e. prevention of FI, during an increase in abdominal pressure under conscious conditions [7]. These data have been mainly documented based on studies of anorectal manometry [4] [6]. Anorectal manometry shows mainly myogenic factors in anorectal functions [20]. There are a few reports on the neurogenic functions of EAS [4] [11] [12]. The regulatory nerve pathway of EAS is the pudendal nerve including both PMN and PSN, which are derived from spinal nerves S2-4 (major regulatory nerves: S3 and S4) [9]. The pudendal nerve projects sensory and motor nerves to the anal canal and EAS, respectively [4]. Therefore, pudendal nerve functions were evaluated using both PMNL and the AMES threshold in this study.

To date, evaluation of the PMN function has focused on the PMN terminal latency [8]-[13]. This latency can reveal unsuspected neuropathy in traumatic FI [13]. In measurements of the PMN terminal latency, stimulation of the PMN is achieved on either side of the pelvis by directing the exploring finger in the anal canal toward the lateral rim of the pelvis, i.e. toward the ischial spine. The laten- 
cy of evoked muscle action responses in EAS (i.e. PMN terminal latency) is recorded after electrical stimulation of PMN in the pelvis [9] [10] [11] [12] [13] [20]. With regard to the PMN terminal latency, artifacts due to pain during evaluations are induced [11] [13]. For investigators employing this technique with inadequate experience, stimulations may not be appropriate. As such, evaluations of the PMN terminal latency may be limited to responses from the ischial spine to EAS, and responses derived from direct stimulations of the EAS muscle may occasionally be evoked [9] [10] [11] [12]. On the other hand, it is possible to use the SMS approach to measure the latency of the whole PMN (the conduction time; extending from the spinal S2-4 motor nerve roots to EAS), and the response is easily induced with high reproducibility, thus providing a reliably consistent index for evaluations [18] [23] [24] [25] [26]. However, to the author's knowledge, there have been no reports of PMNL measurements using the SMS method in patients after LAR with or without FI. Therefore, the present study examined LAR patients with or without FI using SMS.

In the SMS method, values of the PMNL measure the latency time from the stimulation of bilateral sacral nerves to EAS contraction responses. Thus, PMNL values show the total function of the sacral nerve. The normal values of SMS do not indicate laterality differences and are within the range of $3.7-4.4 \pm 0.3-0.5$ ms, where values with a mean + 2SD are considered abnormal [13] [23] [24] [25] [26]. The EAS is regulated by the crisscrossing of the left and right pudendal nerves (PMN and PSN, respectively). In cases where latency is delayed due to nerve injury on either the left or right side, the influence on the EAS muscle is limited as long as the unilateral functions remain intact [6] [18]. As such, certain facilities monitor only the posterior direction of SMS (6 o'clock), which is regulated by the neural crisscrossing of the left and right nerves [10] [18] [23]. Therefore, SMNL of the posterior side may reflect the total function of the right and left sides of PMN. In the present study, to clarify whether there are any differences in PMNL values among the right, left, and posterior sides occur or not in LAR patients, the author measured PMNL of the right, left, and posterior sides of the anal canal. As measurements were conducted by an experienced investigator (Ryouichi Tomita), reproducible normal values with small discrepancies monitored on the right, left, and posterior sites were established in the control subjects. In SMS investigations for LAR patients, marked PMNL prolongation was observed at all sites (right, left, and posterior sites) of patients with compared with those without FI and control subjects. No difference was noted between patients without FI and normal subjects. There was no significant difference among all sides in the results of this study.

Regarding measurement of the latency of sacral and pudendal nerves, the SMS method can measure the latency of both nerves. This is because the SMS procedure stimulates both sacral and pudendal nerves. The muscle contraction response is recorded with a globe-mounted electrode array. The finger bearing this device is inserted into the anal canal so the recording electrode is in direct contact with EAS or PM. Also, contraction of EAS or PM can be easily felt by in- 
serting the index finger into the anal canal. The nerve-stimulation technique using the SMS method can measure the latency in pinpointing both the EAS muscle lesion on the anal canal and the PM muscle lesion at the tip of the anal canal [18] [27]. In addition, in the SMS procedure, LAR patients with FI clearly showed a weak EAS contraction response perceived with the inserted finger compared with those without FI and control subjects. Sato et al. [25] reported that a concentric needle electrode offered precise measurement of the PMNL after SMS. To measure PMNL more accurately, physicians should use a concentric needle electrode for EAS.

In addition, the sensitivity of the anal canal mucosa is important for the prevention of soiling and FI [14] [15] [16] [17] [20]. The anal canal mucosa, which is innervated by PSN, plays an important role in maintaining continence through its sensing of the anal contents [28] [29]. The observation of numerous receptors in the anal canal by Duthie et al. [28] indicated the presence of continence receptors from 2 to $4 \mathrm{~cm}$ above the anal verge. Transitional epithelium in the vicinity of the DL line (i.e. the middle Zone in this study) is well supplied by PSN, connected with sensory terminals and organs, and exhibits the highest sensitivity [14] [15] [16] [28]. Paul et al. [30] concluded that there may be mucosal or submucosal receptors in the distal anal canal at 2 to $4 \mathrm{~cm}$ from the anal verge that support EAS contraction. Fecal continence by EAS is controlled by mucosal or submucosal anal receptors that are regulated by PSN. Lynn et al. [31] reported that PSN to EAS contributes to mechanisms promoting continence and defecation. They concluded that continence is regulated by functional coupling between PMN and PSN. To evaluate the sensitivity of the anal canal, measurement of the AMES threshold is no burden for patients and highly reliable [14] [15] [16] [17]. High-intensity electric currents may evoke nociceptive responses, while low-intensity currents generate abnormal tingling sensations. Measuring the AMES threshold has been preferred because the practical use of the measurement device is simple. The typical AMES threshold varies from 1.5 to $4 \mathrm{~mA}$ [14] [15] [16] [17]. In the present study, the AMES thresholds of control subjects in the upper, middle, and lower zones of the anal canal registered a peak value of $3.1 \mathrm{~mA}$, a finding which was similarly observed in previous studies [14] [15] [16] [17]. The middle zone of the anal canal in control subjects also showed the highest sensitivity, followed by those of the lower and upper zones in that order. In LAR patients with FI, the sensitivities in the upper, middle, and lower zones were less favorable than in patients without FI and control subjects, although differences between patients without FI and control subjects were not significant.

It was also reported that surgery in patients with a narrow pelvis, especially males compared with females, exacerbated bilateral pudendal nerve injury in the anal canal [10] [29]. The EAS is supplied by nerve fibers of the pudendal nerve from S2 to 4 travelling on the levator's undersurface. After that the pudendal nerve leaves the pelvic cavity between the piriformis and coccygeal muscles. Passing the ischial spine, it reaches the ischiorectal fossa, where it lies at the side 
of the obturator muscle within Alcock's canal. Then, the first branch of the pudendal nerve splits to become the inferior rectal and perineal branches [32]. The inferior branch (PMN) controls the motility of EAS, and the perineal branch (PSN) controls the sensory function. PMN and PSN become gradually thiner from the anal to oral side in the anal canal. These two branches directly control EAS [32] [33]. Pfeifer et al. [34] reported that anal sphincter stretching during anastomosis also injured PMN and PSN. Rieger et al. [35] also suggested that the level of anastomosis in patients with FI was much lower than that in patients without FI. Also, the operation, especially anastomosis, was documented as having been much more difficult in patients with compared with without FI. In view of these facts, patients with FI might have suffered more intraoperative damage to the pudendal nerve innervating the EAS muscle than patients without FI. It was reported that both PMN and PSN in LAR patients who undergo anastomosis of $2 \mathrm{~cm}$ or less from the anal verge was easily damaged compared with that of $4 \mathrm{~cm}$ or more [1] [2] [3] [7] [12]. Andrius et al. [6] stated that direct injury is due to the dilatation of the anal canal that is necessary to introduce the stapling device, and the nerves might also be damaged during mobilization of the rectum or following surgery. In the present study, patients after LAR with DAAV of 2 $\mathrm{cm}$ or less $($ Male/Female $=17 / 3)$ showed a tendency toward FI compared with that of $4 \mathrm{~cm}$ or more $($ Male/Female $=23 / 17)$. The former patients with a narrow pelvis experienced more practical difficulties (the intrapelvic procedures and operative interventions through the anus) than the latter patients without a narrow pelvis. As the anal canal was excessively extended by surgeons, injury of the bilateral PMN and PSN in the anal canal may have occurred in patients with FI. Both prolongation of the PMNL values and a reduced AMES threshold may be affected by damage of the peripheral PMN and PSN in the EAS muscle. Therefore, both PMN and PSN injury may lead to EAS dysfunction. Also, this study involved patients with an early postoperative status, at 4 months after LAR. To obtain accurate data, physicians should examine PMNL values and the AMES threshold in patients with a functionally stable status about 1 year or more after LAR.

As mentioned above, SMNL of the posterior side reflects the total function of the right and left sides of PMN. Moreover, DL is well-supplied with sensory terminals and organs, and exhibits the highest sensitivity in the anal canal. Therefore, the authors conducted a correlation test between SMNL in the posterior wall and ASMES of the middle zone in the anal canal. In groups A, B, and C, there were significant correlation coefficients between SMNL and AMES. Namely, prolongation of PMNL and a decrease in the sensitivity of the anal canal in an identical patient group with FI after LAR may be due to both PMN and PSN injuries occurring intraoperatively.

The authors concluded that anastomosis near DL may lead to EAS dysfunction due to an impaired pudendal nerve function. In patients with a narrow pelvis, especially male patients, close attention is necessary to avoid causing any mechanical damage in the anal canal while performing operative procedures. Fur- 
thermore, close attention is necessary when including anal canal tractions in a direction along the course of the pudendal nerve, which follows a path in the vicinity of the ischial spine [31] [33]. From the results of this study on the early postoperative status at 4 months after LAR, FI due to EAS dysfunction in LAR patients may be attributed to injuries of both the motor and sensory branches of the pudendal nerve. However, other factors contributing to postoperative incontinence in LAR patients have also been documented [20] [24] [36]. Moreover, Schraffordt et al. [37] suggested that the pudendal nerve continued distally in the anal canal and showed several branches to PM. Wallner [38] revealed that PM is anatomically a part of the EAS muscle rather than the levator ani muscle based on a cadaveric study. Therefore, it is necessary for physicians to clarify the PM function in patients after LAR with or without FI.

The EAS function could be easily assessed by measuring the squeezing anal pressure by anorectal manometry [4] [5] [6]. However, this preliminary study did not involve anorectal manometry. There are many LAR patients with severe FI who have a normal EAS function with a functionally stable status 1 year or more after LAR [37] [39]. Rieger et al. [35] reported that the PMN terminal latency was significantly correlated with squeezing pressure in patients with FI. In order to demonstrate the EAS function correctly, the maximum anal sphincter pressure during voluntary contraction should be evaluated by anorectal manometry and compared with both SMNL values and the AMES threshold.

This was a retrospective study with a small sample size. An increased number of patients after LAR with or without FI should be performed in the future. This study focused on FI at 4 months after LAR. Regarding PMNL values, the AMES threshold, and anorectal manometry (especially, the maximum squeezing pressure), discussions should also include low anterior resection syndrome such as urgency, increased bowel movements, fluctuation or even constipation with an early postoperative status less than 6 months after LAR.

\section{Conclusion}

FI after LAR with a short DVVA (i.e. anastomosis near DL) may lead to EAS dysfunction due to an impaired pudendal nerve function caused by damage of both PMN and PSN.

\section{Conflicts of Interest}

The authors declare no conflicts of interest regarding the publication of this paper.

\section{References}

[1] Junginger, T., Rassouli, S., Goenner, U., Lollert, A. and Blettner, M. (2014) Correlation between Fecal Incontinence and Quality of Life after Low Anterior Resection for Rectal Cancer. Colorectal Cancer, 3, 17-26. https://doi.org/10.2217/crc.13.77

[2] Keane, C., Wells, C., O’Grady, G. and Bissett, I.P. (2017) Defining Low Anterior Resection Syndrome: A Systematic Review of the Literature. Colorectal Disease, 19, 
713-722. https://doi.org/10.1111/codi.13767

[3] Badic, B., Joumond, A., Thereaux, J., Gancel, C.H. and Bail, J.P. (2018) Long-Term Functional and Oncological Results after Sphincter-Saving Resection for Rectal Cancer: Cohort Study. The International Journal of Surgery, 52, 1-6. https://doi.org/10.1016/j.ijsu.2018.02.003

[4] Craig, H. and Olson, M.D. (2014) Diagnostic Testing for Fecal Incontinence. Clinics in Colon and Rectal Surgery, 27, 85-90. https://doi.org/10.1055/s-0034-1383901

[5] Kim, J.C., Yu, C.S., Lim, S.B., Kim, C.W., Park, I.J. and Yoon, Y.S. (2015) Outcomes of Ultra-Low Anterior Resection Combined with or without Intersphincteric Resection in Lower Rectal Cancer Patients. The International Journal of Colorectal Disease, 30, 1311-1321. https://doi.org/10.1007/s00384-015-2303-x

[6] Audrius, D. and Narimantas, E.S. (2016) Usefulness of Anorectal Manometry for Diagnosing Continence Problems after a Low Anterior Resection. Annals of Coloproctology, 32, 101-104. https://doi.org/10.3393/ac.2016.32.3.101

[7] Nikoletta, D., Othon, M., Dimitrios, M. and John, G. (2015) Low Rectal Cancer: Sphincter Preserving Techniques-Selection of Patients, Techniques and Outcomes. World Journal of Gastrointestinal Oncology, 7, 55-70. https://doi.org/10.4251/wjgo.v7.i7.55

[8] Abdolhasan, T., Mohammad, B., Amin, A. and Alireza, R. (2016) Extended Low Anterior Resection with a Circular Stapler in Patients with Rectal Cancer: A Single Center Experience. Asian Pacific Journal of Cancer Prevention, 16, 8141-8143. https://doi.org/10.7314/APJCP.2015.16.18.8141

[9] Kiff, E.S. and Swash, M. (1995) Slowed Conduction in the Pudendal Nerves in Idiopathic (Neurogenic) Faecal Incontinence. British Journal of Surgery, 71, 614-616. https://doi.org/10.1002/bjs.1800710817

[10] Sangwan, Y.P., Coller, J.A., Barrett, M.S., Murray, J.J., Roberts, P.L. and Schoetz, D.J. (1996) Unilateral Pudendal Neuropathy: Significance and Implications. Diseases of the Colon \& Rectum, 39, 249-251. https://doi.org/10.1007/BF02049459

[11] Tomita, R., Igarashi, S., Ikeda, T., Koshinaga, T., Fujisaki, S. and Tanjoh, K. (2007) Pudendal Nerve Terminal Motor Latency in Patients with or without Soiling 5 Years or More after Low Anterior Resection for Lower Rectal Cancer. World Journal of Surgery, 31, 403-408. https://doi.org/10.1007/s00268-006-0149-7

[12] Yücesoy, A.N., Poçan, S., Cifçi, M., Solmaz, A. and Serefhan, A. (2015) Pudendal Nerve Exposure and Preservation in Low Rectal Surgery by Using Transvaginal Access. Techniques in Coloproctology, 19, 121-123. https://doi.org/10.1007/s10151-014-1253-6

[13] Snooks, S.J., Barnes, P.R., Swash, M. and Henry, M.M. (1985) Damage to the Innervation of the Pelvic Floor Musculature in Chronic Constipation. Gastroenterology, 89, 977-981. https://doi.org/10.1016/0016-5085(85)90196-9

[14] Roe, A.M., Bartolo, D.C. and Mortensen, N.J. (1986) New Method for Assessment of Anal Sensation in Various Anorectal Disorders. British Journal of Surgery, 73, 310-312. https://doi.org/10.1002/bjs.1800730421

[15] Miller, R., Bartolo, D.C., Roe, A., Cervero, F. and Mortensen, N.J. (1988) Anal Sensation and the Continence Mechanism. Diseases of the Colon \& Rectum, 31, 433-438. https://doi.org/10.1007/BF02552612

[16] Tomita, R., Igarashi, S. and Fujisaki, S. (2008) Studies on Anal Canal Sensitivity in Patients with or without Soiling after Low Anterior Resection for Lower Rectal Cancer. Hepatogastroenterology, 55, 1311-1314.

[17] Abe, S., Yoshimoto, T., Yamamoto, M., et al. (2017) Midline Sensory Nerve Supply 
to the Anoscrotal Junction: A Study Using Human Male Fetuses. Okajimas Folia Anatomica Japonica, 94, 17-25. https://doi.org/10.2535/ofaj.94.17

[18] Tomita, R. (2009) Sacral Nerve Function in Patients with Soiling More than 10 Years after Low Anterior Resection for Lower Rectal Cancer. Hepatogastroenterology, 56, 120-123.

[19] Rubin, F., Douard, R. and Wind, P. (2014) The Functional Outcomes of Coloanal and Low Colorectal Anastomoses with Reservoirs after Low Rectal Cancer Resections. The American Surgeon, 80, 1222-1229.

[20] Jorge, J.M.N. and Wexner, S.D. (1993) Etiology and Management of Fecal Incontinence. Diseases of the Colon \& Rectum, 36, 77-97.

https://doi.org/10.1007/BF02050307

[21] Ito, M., Saito, N., Sugito, M., Kobayashi, A., Nishizaawa, Y. and Tsunoda, Y. (2009) A Analysis of Clinical Factors Associated with Anal Function after Intersphincteric Resection for Very Low Rectal Cancer. Diseases of the Colon \& Rectum, 52, 64-70. https://doi.org/10.1007/DCR.0b013e31819739a0

[22] Koda, K., Yasuda, H., Hirano, A., et al. (2009) Evaluation of Postoperative Damage to Anal Sphincter/Levator Ani Muscles with Three-Dimensional Vector Manometry after Sphincter-Preserving Operation for Rectal Cancer. Journal of the American College of Surgeons, 208, 362-367. https://doi.org/10.1016/j.jamcollsurg.2008.10.035

[23] Maccabee, P.J., Lipitz, M.E., Desudchit, T., et al. (1996) A New Method Using Neuromagnetic Stimulation to Measure Conduction Time within the Cauda Equina. Electroencephalography and Clinical Neurophysiology, 101, 153-166. https://doi.org/10.1016/0924-980X(95)00264-L

[24] Pelliccioni, G., Scarpino, O. and Piloni, V. (1997) Motor Evoked Potentials Recorded from External Anal Sphincter by Cortical and Lumbo-Sacral Magnetic Stimulation: Normative Data. Journal of the Neurological Sciences, 149, 69-72. https://doi.org/10.1016/S0022-510X(97)05388-4

[25] Sato, T., Konishi, F. and Kanazawa, K. (2000) Variations in Motor Evoked Potential Latencies in the Anal Sphincter System with Sacral Magnetic Stimulation. Diseases of the Colon \& Rectum, 43, 966-970. https://doi.org/10.1007/BF02237361

[26] Tantiphlachiva, T., Attaluri, A., Valestin, J., Yamada, T. and Rao, S.S. (2011) Translumbar and Transsacral Motor-Evoked Potentials: A Novel Test for Spino-Anorectal Neuropathy in Spinal Cord Injury. American Journal of Gastroenterology, 106, 907-914. https://doi.org/10.1038/ajg.2010.478

[27] Tomita, R. (2014) Neurological Function of the Puborectalis Muscle in Patients with or without Soiling after Ileal J-Pouch-Anal Anastomosis for Ulcerative Colitis in Childhood. Journal of Pediatric Surgery, 49, 1626-1630. https://doi.org/10.1016/j.jpedsurg.2014.07.002

[28] Duthie, H.L. and Bennett, R.C. (1963) The Relation of Sensation in the Anal Canal to the Functional anal Sphincter: A Possible Factor in Anal Continence. Gut, 4, 179-182. https://doi.org/10.1136/gut.4.2.179

[29] Ho, Y.H. and Goh, H.S. (1995) Unilateral Anal Electrosensation. Modified Technique to Improve Quantification of Anal Sensory Loss. Diseases of the Colon \& Rectum, 38, 239-244. https://doi.org/10.1007/BF02055594

[30] Paul, M.A.B., Freddy, M.P. and Jose, B.O. (2013) Fecal Continence Revisited: The Anal External Sphincter Continence Reflex. Diseases of the Colon \& Rectum, 56, 1273-1281. https://doi.org/10.1097/DCR.0b013e3182a42d16

[31] Lynn, P.A. and Brookes, S.J. (2011) Pudendal Afferent Innervation of the Guinea 
Pig External Anal Sphincter. Neurogastroenterology \& Motility, 23, 871-e343. https://doi.org/10.1111/j.1365-2982.2011.01741.x

[32] Matzel, K.E., Schmidt, R.A. and Tanagho, E.A. (1990) Neuroanatomy of the Striated Muscular anal Continence Mechanism. Diseases of the Colon \& Rectum, 33, 666-673. https://doi.org/10.1007/BF02150742

[33] Maldonado, P.A., Chin, K., Garcia, A.A. and Corton, M.M. (2015) Anatomic Variations of Pudendal Nerve within Pelvis and Pudendal Canal: Clinical Applications. American Journal of Obstetrics \& Gynecology, 213, 727.e1-6. https://doi.org/10.1016/j.ajog.2015.06.009

[34] Pfeifer, J., Salanga, V.D., Agachan, F., Weiss, E.G. and Wexner, S.D. (1997) Variation in Pudendal Nerve Terminal Motor Latency According to Disease. Diseases of the Colon \& Rectum, 40, 79-83. https://doi.org/10.1007/BF02055686

[35] Rieger, N.E., Sarre, R.G., Saccone, G.T., Schloithe, A.C. and Wattchow, D.A. (1997) Correlation of Pudendal Nerve Terminal Motor Latency with the Results of Anal Manometry. The International Journal of Colorectal Disease, 12, 303-307. https://doi.org/10.1007/s003840050111

[36] Kupsch, J., Jackisch, T., Matzel, K.E., et al. (2018) Outcome of Bowel Function Following Anterior Resection for Rectal Cancer: An Analysis Using the Low Anterior Resection Syndrome. The International Journal of Colorectal Disease, 33, 787-798. https://doi.org/10.1007/s00384-018-3006-x

[37] Schraffordt, S.E., Tjandra, J.J., Eizenberg, N. and Dwyer, P.L. (2004) Anatomy of the Pudendal Nerve and Its Terminal Branches: A Cadaver Study. ANZ Journal of Surgery, 74, 23-26. https://doi.org/10.1046/j.1445-1433.2003.02885.x

[38] Wallner, C. (2008) Is the Puborectalis Muscle Part of the Levator Ani Muscle? Diseases of the Colon \& Rectum, 51, 1165-1166. https://doi.org/10.1007/s10350-008-9249-9

[39] Ihnat, P., Vavra, P., Prokop, J., Pelikan, A., Ihnat Rudinska, L. and Penkal, I. (2018) Functional Outcome of Low Anterior Resection Evaluated by Anorectal Manometry. ANZ Journal of Surgery, 88, E512-E516. https://doi.org/10.1111/ans.14207 